\title{
Certified Connection Tableaux Proofs for HOL Light and TPTP
}

\author{
Cezary Kaliszyk \\ University of Innsbruck \\ cezary.kaliszyk@uibk.ac.at
}

\author{
Josef Urban \\ Radboud University Nijmegen \\ josef.urban@gmail.com
}

\author{
Jiří Vyskočil \\ Czech Technical University in Prague \\ jiri.vyskocil@gmail.com
}

\begin{abstract}
In recent years, the Metis prover based on ordered paramodulation and model elimination has replaced the earlier built-in methods for general-purpose proof automation in HOL4 and Isabelle/HOL. In the annual CASC competition, the leanCoP system based on connection tableaux has however performed better than Metis. In this paper we show how the leanCoP's core algorithm can be implemented inside HOL Light. leanCoP's flagship feature, namely its minimalistic core, results in a very simple proof system. This plays a crucial role in extending the MESON proof reconstruction mechanism to connection tableaux proofs, providing an implementation of leanCoP that certifies its proofs. We discuss the differences between our direct implementation using an explicit Prolog stack, to the continuation passing implementation of MESON present in HOL Light and compare their performance on all core HOL Light goals. The resulting prover can be also used as a general purpose TPTP prover. We compare its performance against the resolution based Metis on TPTP and other interesting datasets.
\end{abstract}

Categories and Subject Descriptors I.2.3 [Deduction and Theorem Proving]: Inference engines; F.4.1 [Mathematical Logic]: Mechanical theorem proving

Keywords leanCoP, HOL Light, connection tableaux, certified proofs, automated reasoning, interactive theorem proving

\section{Introduction and Related Work}

The leanCoP [26] automated theorem prover (ATP) has an unusually good ratio of performance to its implementation size. While its core algorithm fits on some twenty lines of Prolog, starting with CASC-21 [35] it has regularly beaten Otter [22] and Metis [8] in the FOF division of the CASC ATP competitions. In 2014, leanCoP solved 158 FOF problems in CASC-J7, ${ }^{1}$ while Prover9 [21] solved 95 problems. On the large-theory (chainy) division of the MPTP Challenge benchmark ${ }^{2}$, leanCoP's goal-directed calculus

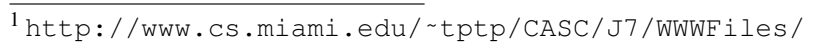
Divisionsummaryl.html

${ }^{2}$ http://www.cs.miami.edu/ tptp/MPTPChallenge/
}

Permission to make digital or hard copies of part or all of this work for personal or classroom use is granted without fee provided that copies are not made or distributed for profit or commercial advantage and that copies bear this notice and the full citation on the first page. Copyrights for third-party components of this work must be honored. For all other uses, contact the owner/author(s).

CPP '15, January 13-14, 2015, Mumbai, India.

Copyright is held by the owner/author(s).

ACM 978-1-4503-3300-9/15/01

http://dx.doi.org/10.1145/2676724.2693176 beats also SPASS 2.2 [41], and further AI-style strengthening by integrating into leanCoP learning-based guidance trained on such larger ITP corpora is an interesting possibility [39].

Compact ATP calculi such as leanTAP [2] and MESON [20] have been used for some time in Isabelle [27, 28] and HOLs [5] as general first-order automation tactics for discharging goals that are already simple enough. With the arrival of large-theory "hammer" linkups [10, 13, 18, 29, 37, 40] between ITPs, state-of-theart ATPs such as Vampire [16] and E [32], and premise selection methods [17], such tactics also became used as a relatively cheap method for reconstructing the (minimized) proofs found by the stronger ATPs. In particular, Hurd's Metis has been adopted as the main proof reconstruction tool used by Isabelle's Sledgehammer linkup [3,30], while Harrison's version of MESON could reconstruct in 1 second about $80 \%$ of the minimized proofs found by $E$ in the first experiments with the HOLYHammer linkup [11].

Since HOL Light already contains a lot of the necessary infrastructure for Prolog-style proof search and its reconstruction, integrating leanCoP into HOL Light in a similar way as MESON should not be too costly, while it could lead to interesting strengthening of HOL Light's first-order automation and proof reconstruction methods. In the main part of this paper (Section 3) we describe how this was done, resulting in an OCaml implementation of leanCoP and a general leanCoP first-order tactic in HOL Light. Section 4 then compares their performance with MESON, Metis and the Prolog version of leanCoP in several scenarios, showing quite significant improvements over MESON and Metis. However, first (Section 2) we have to introduce leanCoP and its calculus.

\section{2. leanCoP and Its Calculus}

leanCoP is an automated theorem prover for classical first-order logic based on a compact Prolog implementation of the clausal connection (tableaux) calculus $[19,26]$ with several simple strategies that significantly reduce the search space on many problems. In contrast to saturation-based calculi used in most of the stateof-the-art ATPs (E, Vampire, etc.), connection calculi implement goal-oriented proof search. Their main inference step connects a literal on the current path to a new literal with the same predicate symbol but different polarity. The formal definition (derived from Otten [25]) of the particular connection calculus relevant in leanCoP is as follows:

Definition 1. [Connection calculus] The axiom and rules of the connection calculus are given in Figure 1. The words of the calculus are tuples " $C, M$, Path" where the clause $C$ is the open subgoal, $M$ is a set of clauses in disjunctive normal form (DNF) transformed from axioms $\wedge$ conjecture with added nullary predicate $\sharp^{3}$

\footnotetext{
${ }^{3}$ We suppose that $\sharp$ is a new predicate that does not occur anywhere in axioms and conjecture.
} 
to all positive clauses ${ }^{4}$, and the active path Path is a subset of a path through $M$. In the rules of the calculus $C, C^{\prime}$ and $C^{\prime \prime}$ are clauses, $\sigma$ is a term substitution, and $L_{1}, L_{2}$ is a connection with $\sigma\left(L_{1}\right)=\sigma\left(\overline{L_{2}}\right)$. The rules of the calculus are applied in an analytic (i.e. bottom-up) way. The term substitution $\sigma$ is applied to the whole derivation.

The connection calculus is correct and complete [19] in the following sense: A first-order formula $M$ in clausal form is valid iff there is a connection proof for " $\neg \sharp, M,\{\}$ ", i.e., a derivation for " $\neg \sharp, M,\{\}$ " in the connection calculus so that all leaves are axioms. The following Prolog predicate prove/5 implements the axiom, the reduction and the extension rule of the basic connection calculus in leanCoP. The code will be explained in more detail later. The comments (gap for ...) in the code represent optimizations that will be filled in and explained in the following subsections.

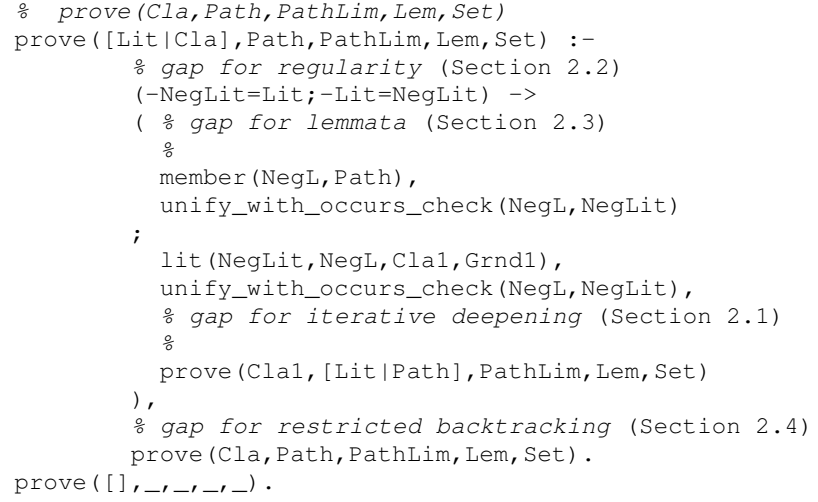

The tuple " $C, M, P a t h$ " in connection calculus is here represented as follows:

- $C$ representing the open subgoal is a Prolog list $\mathrm{Cla}$;

- the active path Path is a Prolog list Path;

- $M$ is written into Prolog's database before the actual proof search starts in a such way that for every clause $C \in M$ and for every literal $C \in M$ the fact $\mathrm{l}$ it (Indexing_L, L, C1, Grnd) is stored, where $\mathrm{C} 1=C \backslash\{\mathrm{L}\}$ and Grnd is $\mathrm{g}$ if $C$ is ground, otherwise Grnd is $n$. Indexing_L is same as $L$ modulo all its variables which are fresh (there is no twice or more occurrences in Indexing_L) everywhere in Indexing_L and it is used for fast finding the right fact in database without affecting the logically correct L by standard Prolog unification without occurs check.

- Atoms are represented by Prolog atoms, negation by “-”.

- The substitution $\sigma$ is stored implicitly by Prolog.

PathLim is the current limit used for iterative deepening, Lem is the list of usable (previously derived) lemmas, Set a list of options, and Proof is the resulting proof. This predicate succeeds (using iterative deepening) iff there is a connection proof for the tuple represented by $\mathrm{Cla}$, the DNF representation of the problem stored in Prolog's database using the lit predicate, and a Path with $\mid$ Path $\mid<$ PathLim where PathLim is the maximum size of the active Path. The predicate works as follows:

Line 18 implements the axiom, line 4 calculates the complement of the first literal Lit in $\mathrm{Cla}$, which is used as the principal literal for the next reduction or extension step. The reduction rule is implemented in lines 7,8 and 17. At line 7 and 8 it is checked whether the active path Path contains a literal NegL that unifies

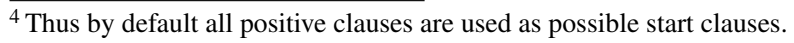

axiom: $\overline{\{\}, M, \text { Path }}$

reduction rule: $\frac{C, M, P a t h \cup\left\{L_{2}\right\}}{C \cup\left\{L_{1}\right\}, M, P a t h \cup\left\{L_{2}\right\}}$

where there exists a unification substitution $\sigma$ such that $\sigma\left(L_{1}\right)=\sigma\left(\overline{L_{2}}\right)$

extension rule: $\frac{C^{\prime} \backslash\left\{L_{2}\right\}, M, \text { Path } \cup\left\{L_{1}\right\} \quad C, M, \text { Path }}{C \cup\left\{L_{1}\right\}, M, \text { Path }}$

where $C^{\prime}$ is a fresh copy of some $C^{\prime \prime} \in M$ such that $L_{2} \in$ $C^{\prime}$ and $\sigma\left(L_{1}\right)=\sigma\left(\overline{L_{2}}\right)$ where $\sigma$ is unification substitution.

Note that the $\sigma$ used in the reduction and extension rules must be applied on all literals in all derivations except the literals in the set $M$ because these literals are not affected by any substitution $\sigma$.

Figure 1. The basic clause connection calculus used in leanCoP.

with the complement NegLit of the principal literal Lit. In this case the alternative lines after the semicolon are skipped and the proof search for the premise of the reduction rule is invoked in line 17. The extension rule is implemented in lines 10, 11, 14 and 17. Lines 10 and 11 are used to find a clause that contains the complement NegLit of the principal literal Lit. Clal is the remaining set of literals of the selected clause and the new open subgoal of the left premise. The proof search for the left premise of the extension rule, in which the active path Path is extended by the principal literal Lit, is invoked in line 14, and if successful, we again continue on line 17.

Compared with standard tableaux or some sequent calculi, connection calculi are not confluent. ${ }^{5}$ To achieve completeness, an extensive use of backtracking is required. leanCoP uses two simple incomplete strategies (namely options scut and cut) for restricting backtracking that significantly reduces the search space [25] without affecting the ability to find proofs in most tested cases (see Section 4).

Another major problem in connection calculi is the integration of equality. The paramodulation method that is widely used in saturation-based ATPs is not complete for goal-oriented approach of connection calculi. Therefore equality in leanCoP and similar ATPs is usually managed by adding the axioms of equality (reflexivity, symmetry, transitivity and substitutivity).

To obtain the clausal form, leanCoP uses its own implementation of clausifier introducing definitions (the def option), which seems to perform better with leanCoP's core prover than other standard clausifiers (TPTP2X using the option -t clausify: tptp, FLOTTER [23] and E) or direct transformation into clausal form (nodef option in leanCoP) [25]. In the following subsections, we summarize several further methods used by leanCoP that improve its performance.

\subsection{Iterative deepening}

Prolog uses a simple incomplete depth-first search strategy to explore the search space. This kind of incompleteness would result in a calculus that hardly proves any formula. In order to obtain a complete proof search in the connection calculus, iterative deepening on the proof depth, i.e. the size of the active path, is performed. It is achieved by inserting the following lines into the code:

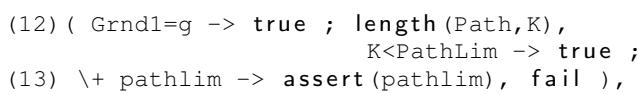

where the Prolog predicate $\backslash+$ Goal succeeds only if Goal fails (negation as failure). The whole prover runs in the following itera-

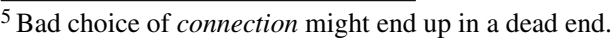


tive sense starting from PathLimit $=1$ :

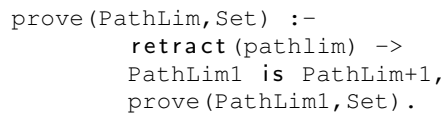

When the extension rule is applied and the new clause is not ground, i.e. it does not contain any variable, it is checked whether the size $\mathrm{K}$ of the active path exceeds the current path limit PathLim (line 12). In this case the dynamic predicate path $\mathrm{lim} / 0$ is written into the Prolog's database (line 13) indicating the need to increase the path limit if the proof search with the current path limit fails. If the proof search fails and the predicate pathlim can be found in the database, then PathLim is increased by one and the proof search starts again.

\subsection{Regularity Condition Optimization}

DEFINITION 2. A connection proof is regular iff no literal occurs more than once in the active path.

Since the active path corresponds to the set of literals in a branch in the connection tableau representation, a connection tableau proof is regular if in the current branch no literal occurs more than once. The regularity condition is integrated into the connection calculus in Figure 1 by imposing the following restriction on the reduction and extension rule: $\forall L^{\prime} \in C \cup\left\{L_{1}\right\}: \sigma\left(L^{\prime}\right) \notin \sigma($ Path $)$

LEMmA 2.1. A formula $M$ in clausal form described above is valid iff there is a regular connection proof for “ $\neg \sharp, M,\{\}$ ”

Regularity is correct, since it only imposes a restriction on the applicability of the reduction and extension rules. The completeness proof can be found in $[19,26]$. The regularity condition must be checked whenever the reduction, extension or lemma rule is applied. The substitution $\sigma$ is not modified, i.e. the regularity condition is satisfied if the open subgoal does not contain a literal that is syntactically identical with a literal in the active path. This is implemented by inserting the following line into the code:

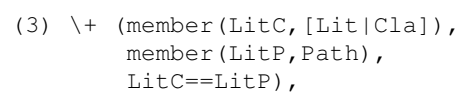

Here the code inside negation succeeds if the open subgoal [Lit $\mid \mathrm{Cla}$ ] contains a literal LitC that is syntactically identical (built-in predicate $==/ 2$ in Prolog) with a literal LitP in the active path $\mathrm{Path}$. The built-in predicate member $/ 2$ is used to enumerate all elements of a list.

\subsection{Lemmata optimization}

The set of lemmata is represented by the list Lem. The lemma rule is implemented by inserting the following lines:

(5) ( member(LitL, Lem), Lit==LitL

(6) ;

In order to apply the lemma rule, the substitution $\sigma$ is not modified, i.e. the lemma rule is only applied if the list of lemmata Lem contains a literal Lit L that is syntactically identical with the literal Lit. Furthermore, the literal Lit is added to the list Lem of lemmata in the (left) premise of the reduction and extension rule by adapting the following line:

(15) prove (Cla,Path,PathLim, [Lit|Lem], Set).

In the resulting implementation, the lemma rule is applied before the reduction and extension rules.

\subsection{Restricted backtracking}

In Prolog the cut (!) is used to cut off alternative solutions when Prolog tries to prove a goal. The Prolog cut is a built-in predicate, which succeeds immediately when first encountered as a goal. Any attempt to re-satisfy the cut fails for the parent goal, i.e. other alternative choices are discarded that have been made from the point when the parent goal was invoked. Consequently, restricted backtracking is achieved by inserting a Prolog cut after the lemma, reduction, or extension rule is applied. It is implemented by inserting the following line into the code:

(16) ( member (cut, set) $\rightarrow$ ! ; true),

Restricted backtracking is switched on if the list Set contains the option cut. The restricted start step is used if the list Set includes the option scut. In this case only the first matching clause to starting $\neg \sharp$ literal is used. Restricted backtracking and restricted start step lead to an incomplete proof search. In order to regain completeness, these strategies can be switched off when the search reaches a certain path limit. If the list Set contains the option comp (Limit), where Limit is a natural number, the proof search is stopped and started again without using incomplete search strategies.

\section{OCaml Implementation}

In this section, we first discuss our implementation ${ }^{6}$ of leanCoP in OCaml and its integration in HOL Light: the transformation of the higher-order goal to first order and the proof reconstruction. After that we compare our implementation to Harrison's implementation of MESON.

\section{1 leanCoP in OCaml}

Otten's implementation of leanCoP uses the Prolog search, backtracking, and indexing mechanisms to implement the connection tableaux proof search. This is a variation of the general idea of using the "Prolog technology theorem prover" (PTTP) proposed by Stickel [33], in which connection tableaux takes a number of advantages from its similarity to Prolog.

In order to implement an equivalent program in a functional programming language, one needs to use either an explicit stack for keeping track of the current proof state (including the trail of variable bindings), or continuation passing style. We choose to do the former, namely we add an explicit todo (stack), subst (trail) and off (offset in the trail) arguments to the main prove function. The stack keeps a list of tuples that are given as arguments to the recursive invocations of prove, whose full OCaml declaration (taking the open subgoal as its last argument) then looks as follows:

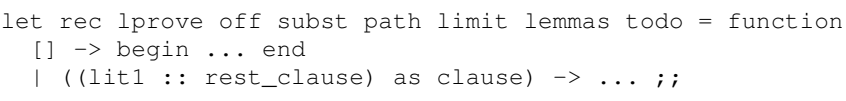

The function performs the proof search to the given depth, and if a proof has not been found, it returns unit. It takes special attention to traverse the tree in the same order as the Prolog version. In particular, when the global option "cut" (restricting backtracking) is off, it performs all the backtrackings explicitly, while if "cut" is on, the parts of backtracking avoided in Prolog are also omitted. When a proof is found, the exception 'Solved' is raised: no further search is performed and the function exits with this exception.

The OCaml version and the Prolog version (simplified and with symbols renamed for clarity of comparison) are displayed together in Fig. 2. The algorithm proceeds as follows:

\footnotetext{
${ }^{6}$ Available online at http://cl-informatik.uibk.ac.at/ cek/cpp15
} 
literals, the two can be used interchangeably in the proof procedure. We start the FOL algorithm by trying to prove $\neg \sharp$.

Since the final leanCoP proof may include references to lemmas, the reconstruction cannot be performed the same way as it is done in MESON. There, a tree structure is used for finished proofs. Each subgoal either closes the branch (the literal is a negation of a literal already present on the path) or is a branch extension with a (possibly empty) list of subgoals. In leanCoP, each subgoal can refer to previous subgoals, so the order of the subgoals becomes important. We therefore flatten the tree to a list, which needs to be traversed in a linear order to reconstruct the proof.

We define a type of proof steps, one for each proof step in the calculus. Each application of a lemma step or path step constructs a proof step with an appropriate first-order term. For an application of a tableaux extension step we use Harrison's contrapositive mechanism: we store the reference to the actual transformed HOL theorem whose conclusion is a disjunction together with the number of the disjunct that got resolved. ${ }^{7}$

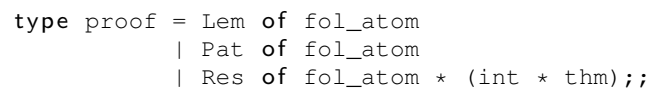

A list of such proof steps together with a final substitution and an initially empty list of already proved lemmas are the complete input to the proof reconstruction procedure. The reconstruction procedure always looks at the first step on the list. First, a HOL term is constructed from the FOL term with the final substitution applied. This step is straightforward, as it amounts to reversing the mapping of variables and constants applied to transform the HOL CNF to FOL CNF, with new names invented for new variables. Next, we analyze the kind of the step. If the step is a path step, the theorem $t m \vdash t m$ is returned, using the HOL ASSUME proof rule. If the step is a lemma step, the theorem whose conclusion is equal to $t m$ is found on the list of lemmas and returned. Finally, if the proof step is an extension step, we first find the disjuncts of the HOL theorem in the proof step apart from the one that got matched. We then fold over this list, at every step calling the reconstruction function recursively with the remaining proof steps and the list of lemmas extended by each of the calls. The result of the fold is the list of theorems $\left[\vdash t m_{1}, \vdash t m_{2}, \ldots, \vdash t m_{n}\right]$ which gets matched with the contrapositive theorem $\vdash t m_{1} \wedge \ldots \wedge t m_{n} \rightarrow t m_{g}$ using the HOL proof rule MATCH_MP to obtain the theorem $\vdash t m_{g}$. Finally, by matching this theorem to the term $t m$ the theorem $\vdash t m$ is obtained.

As the reconstruction procedure traverses the list, it produces the theorem that corresponds to the first goal, namely ... $\vdash \neg \sharp$. By unfolding the definition of $\sharp$, we obtain $\ldots \vdash \perp$ which concludes the refutation proof.

\subsection{Comparison with MESON}

The simplified OCaml code of the core HOL Light's MESON algorithm as described in [6] is as follows:

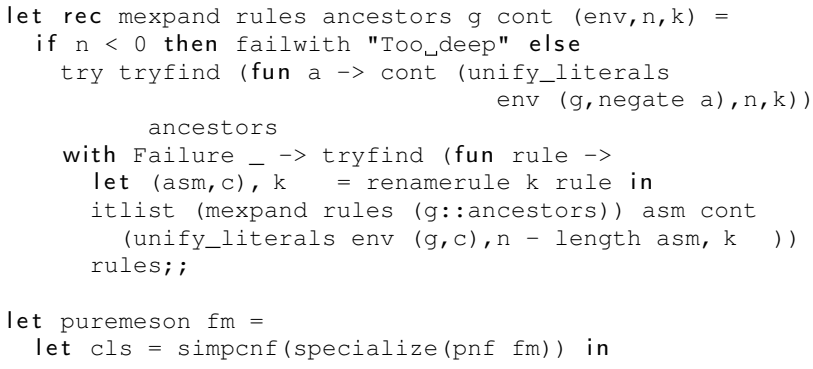

${ }^{7}$ Contrary to the name, the HOL Light type fol_atom implements a literal: it is either positive or negative.

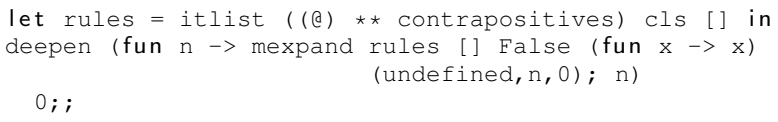

The toplevel puremeson function proceeds by turning the input formula into a clausal form, making contrapositives (rules) from the clauses, and then repeatedly calling the mexpand function with these rules using iterative deepening over the number of nodes permitted in the proof tree.

The mexpand function takes as its arguments the rules, an (initially empty) list of goal ancestors, the goal $g$ to prove (initially False, which was also added to all-negative clauses when creating contrapositives), a continuation function cont for solving the rest of the subgoals (initially the identity), and a tuple consisting of the current trail env, the number $\mathrm{n}$ of additional nodes in the proof tree permitted, and a counter $\mathrm{k}$ for variable renaming.

If the allowed node count is not negative, mexpand first tries to unify the current goal with a negated ancestor, followed by calling the current continuation (trying to solve the remaining goals) with the extended trail. If all such unification/continuation attempts fail (i.e., they throw Failure), an extension step is tried with all rules. This means that the head of a (renamed) rule is unified with the goal g, the goal is appended to the ancestors and the mexpand is called (again using list folding with the subsequently modified trail and continuation) for all the assumptions of the rule, decreasing the allowed node count for the recursive calls.

The full HOL Light version of MESON additionally uses a smarter (divide-and-conquer) policy for the size limit, checks the goal for being already among the ancestors, caches continuations, and uses simple indexing. Below we enumerate some of the most important differences between the leanCoP algorithm and MESON and their implementations in HOL Light. Their practical effect is measured in Section 4.

- leanCoP computes and uses lemmas. The literals that correspond to closed branches are stored in a list. Each call to the main prove function additionally looks for the first literal in the list of lemmas. This can cost a linear number of equality checks if no parts of the proof are reused, but it saves computations if there are repetitions.

- Both algorithms use iterative deepening; however the depth and termination conditions are computed differently.

- MESON is implemented in the continuation passing style, so it can use an additional optimization: caching of the continuations. If any continuations are repeated (at the same depth level), the subproof is not retried. Otten's leanCoP uses a direct Prolog implementation which cannot (without further tricks) do such repetition elimination. The implementation of leanCoP in OCaml behaves the same.

- leanCoP may use cut after the lemma step, path step or successful branch closing in the extension step. Implementing this behaviour in OCaml exactly requires multiple Cut exceptions - one for each depth of the proof.

- The checking for repetitions is done in a coarser way in MESON than in leanCoP, allowing leanCoP to skip some work done by MESON.

- The search is started differently in leanCoP and in MESON. leanCoP starts with a conjecture clause, which likely contributes to its relatively good performance on larger problems. 


\section{Experimental Setup and Results}

For the experiments we use HOL Light SVN version 199 (September 2014), Metis 2.3, and leanCoP 2.1. Unless noted otherwise, the systems are run on a 48-core server with AMD Opteron 61742.2 GHz CPUs, 320 GB RAM, and 0.5 MB L2 cache per CPU. Each problem is always assigned one CPU.

The systems are compared on several benchmarks, corresponding to different modes of use: goals coming from HOL Light itself, the general set of problems from the TPTP library, and the large-theory problems extracted from Mizar [36]. The first set of problems is important; however, since these problems come from HOL Light itself, they are likely naturally biased towards MESON. The Mizar problems come from the two MPTP-based benchmarks: the MPTP Challenge and MPTP2078 [1]. These are large-theory problems coming from a different ITP, hence they do not introduce the implicit bias as the HOL Light problems, while coming from a more relevant application domain than the general TPTP problems.

For HOL Light, we evaluate (with 5 second time limit) on two sets of problems. First, we look at 872 MESON-solved HOL Light goals that were made harder by removing splitting. In this scenario the tactic is applied to a subgoal of a proof, which is a bit similar to the Judgement-Day [3] evaluation used for Isabelle/Sledgehammer, where the goals are however restricted to the solvable ones. Table 1 shows the results. Second, we evaluate on the top-level goals (with their dependencies already minimized) that have been solved with the HOLYHammer system [14], i.e., by using the strongest available ATPs. This set is important because tactics such as MESON, Metis and now also leanCoP can be tried as a first cheap method for reconstructing the proofs found by the stronger ATPs. The results are shown in Table 2. In both cases, the OCaml implementation of leanCoP performs best, improving the MESON's performance in the first case by about $11 \%$, and improving on Metis on the second set of problems by about $45 \%$.

Table 3 shows the results of the evaluation on all 7036 FOF problems coming from TPTP 6.0.0, using 10 second time limit. Here the difference to Metis is not so significant, probably because Metis implements ordered paramodulation, which is useful for many TPTP problems containing equality. The improvement over MESON is about $17 \%$. Table 4 and Table 5 show the results on the small (heuristically minimized) and large MPTP Challenge problems. The best version of the OCaml implementation of leanCoP improves by $54 \%$ on Metis and by $90 \%$ on MESON on the small problems, and by $88 \%$ on Metis and $100 \%$ on MESON on the large problems. Here the goal directedness of leanCoP is probably the main factor.

Finally, to get a comparison also with the best ATPs on a larger ITP-oriented benchmark (using different hardware), we have done a 10s evaluation of several systems on the newer MPTP2078 benchmark (used in the 2012 CASC@ Turing competition), see Table 6 and Table 7. The difference to Metis and MESON on the small problems is still quite significant (40\% improvement over MESON), while on the large problems the goal-directedness again shows even more (about 90\% improvement). While Vampire's (version 2.6) SInE heuristic [7] helps a lot on the larger problems [38], the difference there between E (1.8) and our version of leanCoP is not so great as one could imagine given the several orders of magnitude difference in the size of their implementations.

\section{Conclusion and Future Work}

We have implemented an OCaml version of the leanCoP compact connection prover, and the reconstruction of its proofs inside $\mathrm{HOL}$ Light. This proof-reconstruction functionality can be also used to certify in HOL Light an arbitrary TPTP proof produced by lean$\mathrm{CoP}$, thus turning leanCoP into one of the few ATPs whose proofs

\begin{tabular}{lll}
\hline Prover & Theorem $(\%)$ & Unique \\
\hline mlleancop-cut-comp & $759(87.04)$ & 2 \\
mlleancop-nocut & $759(87.04)$ & 2 \\
plleancop-cut & $752(86.23)$ & 0 \\
plleancop-nc & $751(86.12)$ & 0 \\
metis-23 & $708(81.19)$ & 26 \\
meson & $683(78.32)$ & 4 \\
\hline any & $832(95.41)$ & \\
\hline
\end{tabular}

Table 1. Core HOL Light MESON calls without splitting (872 goals), $5 \mathrm{~s}$ per goal

\begin{tabular}{lll}
\hline Prover & Theorem $(\%)$ & Unique \\
\hline mlleancop-cut-comp & $1178(75.70)$ & 12 \\
mlleancop-nocut & $1162(74.67)$ & 0 \\
meson & $1110(71.33)$ & 39 \\
plleancop-nc & $1085(69.73)$ & 0 \\
plleancop-cut & $1084(69.66)$ & 0 \\
metis-23 & $814(52.31)$ & 16 \\
\hline any & $1260(80.97)$ & \\
\hline
\end{tabular}

Table 2. HOL Light dependencies (1556 goals, $5 \mathrm{~s}$ )

\begin{tabular}{lll}
\hline Prover & Theorem (\%) & Unique \\
\hline mlleancop-cut-conj & $1669(23.72)$ & 73 \\
plleancop-cut-conj & $1648(23.42)$ & 21 \\
plleancop-cut & $1622(23.05)$ & 34 \\
mlleancop-cut & $1571(22.32)$ & 9 \\
metis-23 & $1562(22.20)$ & 261 \\
meson & $1430(20.32)$ & 28 \\
plleancop-nocut & $1358(19.30)$ & 25 \\
mlleancop-nocut & $1158(16.45)$ & 3 \\
\hline any & $2433(34.57)$ & \\
\hline
\end{tabular}

Table 3. ТРТР (7036 goals with at least one conjecture, $10 \mathrm{~s}$ )

\begin{tabular}{lll}
\hline Prover & Theorem $(\%)$ & Unique \\
\hline pllean-cut-conj & $103(40.87302)$ & 2 \\
pllean-cut & $99(39.28571)$ & 8 \\
mlleancop-cut-conj & $91(36.11111)$ & 2 \\
mlleancop-cut & $79(31.34921)$ & 0 \\
mlleancop-nocut & $76(30.15873)$ & 0 \\
pllean-nc & $62(24.60317)$ & 1 \\
metis-23 & $59(23.41270)$ & 3 \\
meson-infer & $48(19.04762)$ & 0 \\
\hline any & $124(49.20635)$ & \\
\hline
\end{tabular}

Table 4. Bushy (small) MPTP Challenge problems (252 in total), $10 \mathrm{~s})$ 


\begin{tabular}{lll}
\hline Prover & Theorem $(\%)$ & Unique \\
\hline pllean-cut-conj & $61(24.20635)$ & 5 \\
mlleancop-cut-conj & $60(23.80952)$ & 9 \\
pllean-cut & $57(22.61905)$ & 4 \\
mlleancop-nocut & $47(18.65079)$ & 0 \\
mlleancop-cut & $47(18.65079)$ & 0 \\
metis-23 & $32(12.69841)$ & 3 \\
meson-infer & $30(11.90476)$ & 0 \\
pllean-nc & $26(10.31746)$ & 0 \\
\hline any & $83(32.93651)$ & \\
\hline
\end{tabular}

Table 5. Chainy (large) MPTP Challenge problems (252 in total), $10 \mathrm{~s})$

\begin{tabular}{ll}
\hline Prover & Theorem $(\%)$ \\
\hline Vampire & $1198(57.65)$ \\
e18 & $1022(49.18)$ \\
mlleancop-cut-conj & $613(29.49)$ \\
pllean-cut-conj & $597(28.72)$ \\
metis-23 & $564(27.14)$ \\
mlleancop-cut & $559(26.90)$ \\
pllean-cut & $544(26.17)$ \\
pllean-comp7 & $539(25.93)$ \\
mlleancop-nocut & $521(25.07)$ \\
pllean-nc & $454(21.84)$ \\
meson-infer & $438(21.07)$ \\
any & $1277(61.45)$ \\
\hline
\end{tabular}

Table 6. Bushy (small) MPTP2078 problems (2078 in total), 10 s)

\begin{tabular}{ll}
\hline Prover & Theorem $(\%)$ \\
\hline Vampire & $634(30.51)$ \\
e18 & $317(15.25)$ \\
mlleancop-cut-conj & $243(11.69)$ \\
pllean-cut-conj & $196(9.43)$ \\
pllean-cut & $170(8.18)$ \\
pllean-comp7 & $159(7.65)$ \\
mlleancop-nocut & $150(7.21)$ \\
mlleancop-cut & $146(7.02)$ \\
meson-infer & $145(6.97)$ \\
metis-23 & $138(6.64)$ \\
pllean-nc & $126(6.06)$ \\
any & $693(33.34)$ \\
\hline
\end{tabular}

Table 7. Chainy (large) MPTP2078 problems (2078 in total), $10 \mathrm{~s}$ )

enjoy LCF-style verification in one of the safest LCF-based systems. The performance of the OCaml version on the benchmarks is comparable to the Prolog version, while it always outperforms Metis and MESON, sometimes very significantly on the relevant ITP-related benchmarks.

We provide a $\mathrm{HOL}$ Light interface that is identical to the one offered by MESON, namely we provide two tactics and a rule. LEANCOP_TAC and ASM_LEANCOP_TAC are given a list of helper theorems, and then try to solve the given goal together (or the given goal and assumptions, respectively). The LEANCOP rule, given a list of helper theorems acts as a conversion, i.e., given a term statement it tries to prove a theorem whose conclusion is identical to that of the term. The benchmarks show that these are likely the strongest single-step proof-reconstructing first-order tactics available today in any ITP system. The compatibility with MESON also means that the new tactics can readily strengthen larger systems like PRocH [11] that attempt to replay more complicated ATP proofs in HOL Light by multiple calls to such one-step tactics.

Interesting future directions include re-using parts of the OCaml implementation to develop a reasonably strong internal intuitionistic prover for systems like Coq, based on leanCoP's intuitionistic cousin ileanCoP [24], possible porting of the code to Standard ML for ITP systems such as HOL4 and Isabelle and their (upcoming) hammers [4, 29], and also experiments with integrating our fast OCaml-based learning methods $[9,10,12,15]$ to learn internal guidance of leanCoP's inferencing as a replacement for the so far relatively slow externally provided guidance used in the MaLeCoP prototype [39].

\section{Acknowledgments}

We thank the CPP'15 referees for their helpful comments and suggestions. Kaliszyk was supported by the Austrian Science Fund (FWF): P26201, Urban was supported by NWO grant Knowledgebased Automated Reasoning and Vyskočil was supported by the Grant Agency of the Czech Republic under the Project GACR P103/12/1994

\section{References}

[1] J. Alama, T. Heskes, D. Kühlwein, E. Tsivtsivadze, and J. Urban. Premise selection for mathematics by corpus analysis and kernel methods. J. Autom. Reasoning, 52(2):191-213, 2014. ISSN 0168-7433.

[2] B. Beckert and J. Posegga. leanTAP: Lean tableau-based deduction. $J$. Autom. Reasoning, 15(3):339-358, 1995. . URL http: / / dx . doi . org/10.1007/BF00881804.

[3] S. Böhme and T. Nipkow. Sledgehammer: Judgement Day. In J. Giesl and R. Hähnle, editors, IJCAR, volume 6173 of $L N C S$, pages 107-121. Springer, 2010. ISBN 978-3-642-14202-4.

[4] T. Gauthier and C. Kaliszyk. Premise selection and external provers for HOL4. In Certified Programs and Proofs (CPP'15), Lecture Notes in Computer Science. Springer, 2015. . URL http://dx. doi. org/10.1145/2676724.2693173. http://dx.doi.org/ $10.1145 / 2676724.2693173$.

[5] J. Harrison. Optimizing Proof Search in Model Elimination. In M. McRobbie and J. Slaney, editors, Proceedings of the 13th International Conference on Automated Deduction, number 1104 in LNAI, pages 313-327. Springer, 1996.

[6] J. Harrison. Handbook of Practical Logic and Automated Reasoning. Cambridge University Press, 2009. ISBN 978-0-521-89957-4.

[7] K. Hoder and A. Voronkov. Sine qua non for large theory reasoning. In N. Bjørner and V. Sofronie-Stokkermans, editors, CADE, volume 6803 of LNCS, pages 299-314. Springer, 2011. ISBN 978-3-642-22437-9.

[8] J. Hurd. First-order proof tactics in higher-order logic theorem provers. In M. Archer, B. D. Vito, and C. Muñoz, editors, Design and Application of Strategies/Tactics in Higher Order Logics (STRATA 2003), number NASA/CP-2003-212448 in NASA Technical Reports, pages 56-68, Sept. 2003. URL http: //techreports.larc.nasa.gov/ltrs/PDF/ $2003 / \mathrm{cp} / \mathrm{NASA}-2003-\mathrm{cp} 212448$.pdf.

[9] C. Kaliszyk and J. Urban. Stronger automation for Flyspeck by feature weighting and strategy evolution. In J. C. Blanchette and J. Urban, editors, PxTP 2013, volume 14 of EPiC Series, pages 8795. EasyChair, 2013.

[10] C. Kaliszyk and J. Urban. MizAR 40 for Mizar 40. CoRR, abs/1310.2805, 2013.

[11] C. Kaliszyk and J. Urban. PRocH: Proof reconstruction for HOL Light. In M. P. Bonacina, editor, CADE, volume 7898 of $L N C S$, pages 267-274. Springer, 2013. ISBN 978-3-642-38573-5. 
[12] C. Kaliszyk and J. Urban. Learning-assisted theorem proving with millions of lemmas. J. Symbolic Computation, 2014. . http: //dx.doi.org/10.1016/j.jsc.2014.09.032.

[13] C. Kaliszyk and J. Urban. HOL(y)Hammer: Online ATP service for HOL Light. Mathematics in Computer Science, 2014. . http: //dx.doi.org/10.1007/s11786-014-0182-0.

[14] C. Kaliszyk and J. Urban. Learning-assisted automated reasoning with Flyspeck. J. Autom. Reasoning, 53(2):173-213, 2014. . URL http://dx.doi.org/10.1007/s10817-014-9303-3.

[15] C. Kaliszyk, L. Mamane, and J. Urban. Machine learning of Coq proof guidance: First experiments. In T. Kutsia and A. Voronkov, editors, SCSS 2014, volume 30 of EPiC Series, pages 27-34. EasyChair, 2014.

[16] L. Kovács and A. Voronkov. First-order theorem proving and Vampire. In N. Sharygina and H. Veith, editors, $C A V$, volume 8044 of LNCS, pages 1-35. Springer, 2013. ISBN 978-3-642-39798-1.

[17] D. Kühlwein, T. van Laarhoven, E. Tsivtsivadze, J. Urban, and T. Heskes. Overview and evaluation of premise selection techniques for large theory mathematics. In B. Gramlich, D. Miller, and U. Sattler, editors, $I J C A R$, volume 7364 of $L N C S$, pages 378-392. Springer, 2012. ISBN 978-3-642-31364-6.

[18] D. Kühlwein, J. C. Blanchette, C. Kaliszyk, and J. Urban. MaSh: Machine learning for Sledgehammer. In S. Blazy, C. Paulin-Mohring, and D. Pichardie, editors, ITP 2013, volume 7998 of LNCS, pages 3550. Springer, 2013. . URL http://cl-informatik.uibk. ac.at/users/cek/docs/kuehlwein-itp13.pdf.

[19] R. Letz and G. Stenz. Model elimination and connection tableau procedures. In Robinson and Voronkov [31], pages 2015-2114. ISBN 0-444-50813-9.

[20] D. W. Loveland. Mechanical theorem proving by model elimination. J. the ACM, 15(2):236-251, Apr. 1968.

[21] W. McCune. Prover9 and Mace4. http://www. cs . unm.edu/ mccune/prover9/, 2005-2010.

[22] W. McCune and L. Wos. Otter: The CADE-13 Competition Incarnations. J. Autom. Reasoning, 18(2):211-220, 1997.

[23] A. Nonnengart and C. Weidenbach. Computing small clause normal forms. In Robinson and Voronkov [31], pages 335-367. ISBN 0-44450813-9.

[24] J. Otten. Clausal connection-based theorem proving in intuitionistic first-order logic. In B. Beckert, editor, Automated Reasoning with Analytic Tableaux and Related Methods, International Conference, TABLEAUX 2005, Koblenz, Germany, September 14-17, 2005, Proceedings, volume 3702 of Lecture Notes in Computer Science, pages 245-261. Springer, 2005. ISBN 3-540-28931-3. . URL http://dx.doi.org/10.1007/11554554_19.

[25] J. Otten. Restricting backtracking in connection calculi. AI Commun., 23(2-3):159-182, 2010. . URL http: / / dx. doi . org/10. 3233 /AIC-2010-0464.

[26] J. Otten and W. Bibel. leanCoP: lean connection-based theorem proving. J. Symb. Comput., 36(1-2):139-161, 2003.

[27] L. C. Paulson. Automated reasoning and its applications. chapter Generic Automatic Proof Tools, pages 23-47. MIT Press, Cambridge, MA, USA, 1997. ISBN 0-262-22055-5.
[28] L. C. Paulson. A generic tableau prover and its integration with Isabelle. J. UCS, 5(3):73-87, 1999.

[29] L. C. Paulson and J. Blanchette. Three years of experience with Sledgehammer, a practical link between automated and interactive theorem provers. In 8th IWIL, 2010. Invited talk.

[30] L. C. Paulson and K. W. Susanto. Source-level proof reconstruction for interactive theorem proving. In K. Schneider and J. Brandt, editors, TPHOLs, volume 4732 of LNCS, pages 232-245. Springer, 2007. ISBN 978-3-540-74590-7.

[31] J. A. Robinson and A. Voronkov, editors. Handbook of Automated Reasoning (in 2 volumes). Elsevier and MIT Press, 2001. ISBN 0444-50813-9.

[32] S. Schulz. System description: E 1.8. In K. L. McMillan, A. Middeldorp, and A. Voronkov, editors, Logic for Programming, Artificial Intelligence, and Reasoning - 19th International Conference, LPAR19, Stellenbosch, South Africa, December 14-19, 2013. Proceedings, volume 8312 of Lecture Notes in Computer Science, pages 735-743. Springer, 2013. ISBN 978-3-642-45220-8. . URL http: // dx . doi.org/10.1007/978-3-642-45221-5_49.

[33] M. E. Stickel. A Prolog Technology Theorem Prover: Implementation by an extended Prolog compiler. J. Autom. Reasoning, 4(4):353-380, 1988. . URL http://dx.doi.org/10.1007/BF00297245.

[34] T. Ströder, F. Emmes, P. Schneider-Kamp, J. Giesl, and C. Fuhs. A linear operational semantics for termination and complexity analysis of ISO Prolog. In G. Vidal, editor, LOPSTR, volume 7225 of LNCS, pages 237-252, 2012. .

[35] G. Sutcliffe. The CADE-21 automated theorem proving system competition. AI Commun., 21(1):71-81, 2008. URL http://iospress.metapress.com/content/ q3717606w4338313/.

[36] J. Urban. MPTP 0.2: Design, implementation, and initial experiments. J. Autom. Reasoning, 37(1-2):21-43, 2006.

[37] J. Urban and J. Vyskočil. Theorem proving in large formal mathematics as an emerging AI field. In M. P. Bonacina and M. E. Stickel, editors, Automated Reasoning and Mathematics: Essays in Memory of William McCune, volume 7788 of LNAI, pages 240-257. Springer, 2013. .

[38] J. Urban, K. Hoder, and A. Voronkov. Evaluation of automated theorem proving on the Mizar Mathematical Library. In ICMS, pages 155-166, 2010.

[39] J. Urban, J. Vyskočil, and P. Štěpánek. MaLeCoP: Machine learning connection prover. In K. Brünnler and G. Metcalfe, editors, TABLEAUX, volume 6793 of LNCS, pages 263-277. Springer, 2011. ISBN 978-3-642-22118-7.

[40] J. Urban, P. Rudnicki, and G. Sutcliffe. ATP and presentation service for Mizar formalizations. J. Autom. Reasoning, 50:229-241, 2013. .

[41] C. Weidenbach, B. Afshordel, U. Brahm, C. Cohrs, T. Engel, E. Keen, C. Theobalt, and D. Topić. System description: SPASS version 1.0.0. In Automated Deduction - CADE-16, volume 1632 of LNCS, pages 378-382. Springer Berlin Heidelberg, 1999. ISBN 978-3-540-662228. . URL http://dx.doi.org/10.1007/3-540-48660-7_ 34. 